\title{
Modifications homéorhétiques produites par un $\beta$-agoniste dans les tissus musculaires et adipeux chez le rat
}

\author{
MP Portillo ${ }^{*}$, JA Martinez 2, J Larralde 2 \\ 'Université Paul-Sabatier, faculté de médecine, \\ laboratoire de pharmacologie médicale et clinique, 31000 Toulouse, France; \\ 2 Université de Navarre, département de physiologie et nutrition, 31008 Pamplune, Espagne
}

(Reçu le 22 novembre 1990; accepté le 24 avril 1991)

Résumé - Un agoniste $\beta$-adrénergique non sélectif, le métaprotérénol, administré par voie souscutanée $\left(2 \mathrm{mg} \cdot \mathrm{kg}^{-1} \cdot \mathrm{j}^{-1}\right)$ pendant $23 \mathrm{j}$, induit une modification de la composition corporelle chez le rat. À cause de son effet "répartiteur", il produit une augmentation de la masse protéique de la carcasse $(P<0,05)$ et une diminution des dépôts adipeux $(P<0,01)$. Celle-ci s'accompagne d'un accroissement de la consommation d'oxygène dans le tissu adipeux scapulaire $(P<0,05)$ chez les animaux traités. Les résultats montrent que l'accroissement de la masse musculaire est dû à une diminution de la dégradation des protéines, mise en évidence par la diminution de l'activité de la cathepsine A plutôt qu'à un effet sur le processus de synthèse, mesuré par l'incorporation de phénylalanine tritiée. Les résultats obtenus suggèrent une possible implication de la prostaglandine $E_{2}$ et de l'état rédox, comme médiateurs dans le mécanisme d'action des agonistes $\beta$-adrénergiques.

métaprotérénol / homéorhesis / croissance / composition corporelle / métabolisme protéique

Summary - Homeorhetic changes produced by a $\beta$-agonist on adipose and muscle tissues in rat. A repartitioning effect was observed in young rats after a chronic treatment with a nonselective $\beta$-agonist administered subcutaneously. An increase in carcass protein $(P<0.05)$ accompanied by a reduction in fat stores $(\mathrm{P}<0.01)$ and an increase in back fat oxygen consumption $(P<0.05)$ were found in treated animals. The muscle mass accretion should be attributed to a reduction in muscle protein degradation, based on lower activity of the proteolytic enzyme cathepsin $A$ in treated rats, rather than to changes in protein synthesis, assessed by an amino-acid incorporation technique. The anabolic actions of this compound apparently involve changes in muscle prostaglandine $E_{2}$ and reduction-oxidation state.

metaproterenol / homeorhetic change / growth / body composition / protein metabolism

\footnotetext{
* Correspondance et tirés à part : MP Portillo, departamento de nutrición y bromatología, facultad de farmacia, universidad del País Vasco, Portal de Lasarte s/n, 01007 Vitoria, Espagne.
} 


\section{INTRODUCTION}

Les agonistes $\beta$-adrénergiques reproduisent les effets du système nerveux sympathique (Timmerman, 1987). Leurs actions sont très variées car les récepteurs $\beta$, cibles de cette classe pharmacologique, sont largement distribués dans l'organisme. Parmi les effets métaboliques, on peut signaler la glycogénolyse et la lipomobilisation (Fain et Garcia-Sainz, 1983), de même que l'augmentation de la production de lactate (Hamby et al, 1985) et de la consommation d'oxygène (Landsberg et al, 1984).

Ces agents peuvent être utilisés dans le domaine de la production animale (MacRae et Lobley, 1991). Leurs effets "répartiteurs" provoquent une modification de la composition des carcasses qui correspond à une diminution des dépôts adipeux, associée à un accroissement de la masse protéique (Lafontan et al, 1988). Ces résultats ont été observés chez plusieurs espèces animales : ovins (Baker et al, 1984); porcins (Moser et al, 1986), et bovins (Miller et al, 1988).

D'autres applications, cependant moins claires que les précédentes, à cause d'éventuels effets secondaires indésirables, peuvent être envisagées chez l'homme. On pourrait les employer comme des produits anti-obésité en raison de leur effet lipolytique (Lafontan et al, 1988) et dans le cas des atrophies musculaires, en profitant de leur capacité à induire un accroissement de la masse musculaire (Zeman et al, 1987; Babij et Booth, 1988).

Les agonistes $\beta$-adrénergiques produisent leurs effets par un mécanisme direct impliquant les récepteurs $\beta$ et l'augmentation des taux d'AMPc (Fiems, 1987). Cependant, l'existence de mécanismes indirects, telle la modification des taux plasmatiques de certaines hormones (But- tery et Dawson, 1986), de l'état redox (Tischler et Fagan, 1982) et du flux sanguin (Rothwell et al, 1987), doit être envisagée.

L'étude du mécanisme d'action sur le métabolisme protéique doit être dirigée vers l'évaluation de la synthèse et la dégradation car la quantité de protéines retenues est le résultat du bilan entre les 2 processus (Morgan et al, 1986).

L'objectif de ce travail est tout d'abord d'étudier les effets d'un traitement chronique par un agoniste $\beta$-adrénergique non sélectif, le métaprotérénol, sur la croissance et la composition corporelle des rats jeunes. Les mécanismes d'action susceptibles d'expliquer l'effet anabolique du produit seront précisés.

\section{MATÉRIEL ET MÉTHODES}

\section{Matériel}

Le sulfate de métaprotérénol a été offert par Boehringer-Ingelheim (Barcelone, Espagne). L'acide aminé radioactif, $[2,3,4,5,63 \mathrm{H}]$ Phenylalanine $(100-130 \mathrm{Ci} / \mathrm{mmol})$, employé dans la synthèse protéique et la prostaglandine $E_{2}$ radioactive $\left[5,6,11,12,14,15\right.$ (n) $\left.{ }^{3} \mathrm{H}\right] \mathrm{PGE}_{2}(140-170 \mathrm{Ci} /$ $\mathrm{mmol}$ ), utilisée pour le dosage radioimmunologique, proviennent d'Amersham International (Buckinghamshire, Royaume-Uni). Tous les autres produits ont été fournis par Merck Darmsdat (Francfort, Allemagne).

\section{Animaux}

Des rats Wistar d'un poids initial d'environ $90 \mathrm{~g}$ nourris ad libitum, placés dans des cages à une température de $22^{\circ} \mathrm{C}$ et soumis à des cycles de lumière et d'obscurité de $12 \mathrm{~h}$, ont été utilisés. Le métaprotérénol ( $1 \mathrm{mg} / \mathrm{kg}$ ), a été administré par voie sous-cutanée, 2 fois par jour $(8 \mathrm{~h}$ et $18 \mathrm{~h}$ ) pendant $23 \mathrm{j}$. Les animaux témoins ont reçu 2 injections de sérum physiologique. Au 
moment de l'abattage, les animaux pesaient environ $250 \mathrm{~g}$. Le sang a été recueilli et plusieurs organes ont été pesés et congelés à $-20^{\circ} \mathrm{C}$.

\section{Consommation d'oxygène}

Ce paramètre a été mesuré chez l'animal entier à l'aide d'un respiromètre à volume constant Scholander, dont le fonctionnement s'appuie sur l'enregistrement des variations de pression produites par la respiration de l'animal. La diminution de pression observée traduit la consommation d'oxygène puisque le dioxyde de carbone produit est absorbé par la chaux sodée.

Pour mesurer la consommation d'oxygène des organes isolés, une portion d'environ $0,5 \mathrm{~g}$ du tissu prélevé (adipeux et musculaire) est introduite dans la chambre de l'électrode de Clarke où l'on a mis un milieu de Ringer Looke pour le muscle et de Ringer phosphate pour le tissu adipeux, à $37{ }^{\circ} \mathrm{C}$. Ensuite, on ferme la chambre et on mesure la diminution de la quantité d'oxygène par polarimétrie a:'ec une électrode d'argent-platine et un potentiel de polarisation (650 mV) (Vidaver et Swenson, 1989). Tout ce système est branché à un autre système d'enregistrement graphique qui permet de calculer la quantité d'oxygène disparue du milieu et donc consommée par le tissu.

\section{Acides nucléiques}

La quantité d'acides nucléiques a été mesurée avec deux techniques colorimétriques employant comme réactifs la diphénylamine pour I'ADN et l'orcinol pour l'ARN (Martinez et al, 1987).

\section{Composition corporelle}

Pour la détermination de la composition corporelle, on a préparé, dans un premier temps, une pâte homogène après l'introduction des animaux dans un autoclave et le broyage des carcasses. A partir de cette pâte, on a fait des aliquotes de façon à pouvoir déterminer les teneurs en protéines, graisse, eau et sels minéraux, suivant les indications de I'AOAC (Asso- ciation of Official Analytical Chemists, 1986). La quantité de protéines corporelles a été déterminée par la méthode de Kjeldahl et la quantité de graisse après extraction avec l'éther éthylique. En ce qui concerne la teneur en eau, elle a été mesurée par traitement dans une étuve à $100^{\circ} \mathrm{C}$ jusqu'au moment où le poids de l'échantillon reste constant. Pour mesurer les sels minéraux, on a soumis l'échantillon à une température supérieure à $500^{\circ} \mathrm{C}$ jusqu'à l'obtention de cendres blanches.

\section{Synthèse de protéines}

On prépare une solution mère de phénylalanine froide $150 \mathrm{mmol}^{-1}$ et on ajoute $50 \mu \mathrm{Ci}$ de $\left[{ }^{3} \mathrm{H}\right]$ phénylalanine par $\mathrm{ml}$ de solution mère. Une dose de cette solution $(1 \mathrm{ml} / 100 \mathrm{~g}$ de poids corporel) est administrée par voie intrapéritonéale. Dix minutes après, les animaux sont sacrifiés et les tissus sont prélevés pour mesurer la synthèse protéique. On se sert de la détermination de la quantité de $\left[{ }^{3} \mathrm{H}\right]$ phénylalanine incluse dans les protéines dans les $10 \mathrm{~min}$, selon la technique de Garlick (1980), modifiée par Martinez (1987). Cette valeur nous permet de calculer le taux de synthèse protéique par jour.

On broie $0,3 \mathrm{~g}$ de tissu pour séparer les protéines des acides aminés, de façon à connaître la quantité de radioactivité que l'on trouve dans chaque partie. Le culot de protéines doit être hydrolysé pour faire les déterminations de phénylalanine. La phénylalanine totale doit être transformée en $\beta$-phénétylamine avant d'appliquer la technique fluorimétrique de mesure (Suzuky et Yagi, 1976). La quantité de phénylalanine radioactive est mesurée avec un compteur de radioactivité $\beta$ Beckman LS-2000 et une efficacité de comptage de $30 \%$.

Finalement, on emploie la formule suivante pour faire le calcul :

$$
K s=(S b-100) /(S i-t)
$$

où : Ks : taux de synthèse protéique par jour (\%/ j); $S b$ : radioactivité spécifique dans la protéine (dpm/ $\mu \mathrm{mol}) ; S i$ : radioactivité spécifique libre (dpm/ $\mu \mathrm{mol}$ ); $t$ : temps (approx $10 \mathrm{~min}$ ).

Le $K g$ représente la quantité de protéine retenue tout au long du traitement, exprimée en \%/]. II est obtenu par différence entre la teneur en protéines au début et à la fin du traitement. Le 
$K d$ est calculé indirectement en employant les valeurs de $K g$ et $K s,(K d=K g-K s)$. Le $K_{A R N}$ est le rapport entre la protéine synthétisée par jour et la quantité d'ARN par g de tissu. II est un index de l'activité biosynthétique par unité d'ARN.

\section{Activité de la cathepsine A musculaire}

Un gramme de tissu musculaire est mis en suspension dans une solution contenant $1 \mathrm{ml}$ de EDTA 0,001 mol. I $^{-1}$ et $4 \mathrm{ml}$ de sucrose 0,25 mol..$^{-1}$. Après centrifugation à $1100 \mathrm{~g}$ pendant 10 min a $4{ }^{\circ} \mathrm{C}$, le surnageant est recueilli. L'activité de la cathepsine $A$ est mesurée par colorimétrie en présence de $\mathrm{N}$-carbobenzoxy-Lglutamyl-L-tyrosine (CBZ-Glu-Tyr) et de ninhydrine (Obled et al, 1980). Les unités employées représentent les $\mu \mathrm{mol}$ de tyrosine libérés dans les 30 min d'incubation.

\section{Prostaglandine $E_{2}$}

Un gramme de muscle est mis en suspension dans $5 \mathrm{ml}$ d'alcool froid $\left(0-4^{\circ} \mathrm{C}\right)$. Après centrifugation à $24000 \mathrm{~g}$ pendant $40 \mathrm{~min}$ à $4{ }^{\circ} \mathrm{C}$, le surnageant est prélevé puis déshydraté sous une atmosphère d'azote pendant 1-2 $\mathrm{h}$, à température ambiante. Le culot est remis en suspension dans $2 \mathrm{ml}$ de Tris $(\mathrm{pH}=7,4)$. Finalement, $10 \mu \mathrm{l}$ de cette suspension seront utilisés pour le dosage radio-immunologique de la $\mathrm{PGE}_{2}$ (Levine et Alam, 1979).

On ajoute $0,1 \mathrm{ml} \mathrm{de}\left[{ }^{3} \mathrm{H}\right] \mathrm{PGE}_{2}$ et $0,1 \mathrm{ml}$ d'immuno-sérum de lapin à $0,1 \mathrm{ml}$ de suspension de muscle. Une solution de sérum de lapin et de $\left[{ }^{3} \mathrm{H}\right]$ haptène permet la mesure de la liaison non spécifique. Après une incubation à $37^{\circ} \mathrm{C}$ pendant $1 \mathrm{~h}$, on ajoute $0,1 \mathrm{ml}$ de sérum de lapin et $0,1 \mathrm{ml}$ de sérum de chèvre antilapin. Les immunoprécipités sont centrifugés à $1500 \mathrm{~g}$ pendant $30 \mathrm{~min}$ et on mesure la radioactivité des culots, dissous au préalable par $0,2 \mathrm{ml}$ de $\mathrm{NaOH} 0,1 \mathrm{~N}$ (Warner et al, 1986).

La formule employée pour faire le calcul est la suivante:

$\%$ inhibition $=1-[($ lecture - bruit de fond $)$ / (liaison spécifique-bruit de fond)] $\times 100$.

\section{Lactate et pyruvate}

Le principe de la détermination de ces 2 intermédiaires métaboliques repose sur la mesure spectrophotométrique de la quantité de NADH produite ou consommée dans une réaction enzymatique. Ces 2 mesures ont été faites en employant 2 kits de Boehringer-Mannheim (Barcelone, Espagne).

\section{Activité ATPase $\mathrm{Ca}^{2+}-\mathrm{Mg}^{2+}$}

L'activité de cette enzyme est évaluée par une méthode indirecte. L'activité ATPase est mesurée en présence et en absence de $\mathrm{Ca}^{2+}$; la différence représente l'activité ATPase $\mathrm{Ca}^{2+}-\mathrm{Mg}^{2+}$ (Obejero et al, 1988; Simonides et Van Hardeveld, 1988).

Le phosphore inorganique libéré tout au long de la réaction catalysée par l'ATPase est quantifié par la méthode de Fiske-Subbarow (1925), qui s'appuie sur la réduction du complexe phospho-molybdate par le mélange sulfite-bisulfite.

\section{Traitement statistique}

L'analyse statistique des résultats a été faite en employant le test $t$ de Student. Une valeur de $P<0,05$ est considérée comme significative.

\section{RÉSULTATS}

L'administration de l'agoniste $\beta$-adrénergique, le métaprotérénol (1 $\mathrm{mg} / \mathrm{kg}$ ) par voie sous-cutanée, pendant $23 \mathrm{j}$, ne modifie pas la croissance des animaux, comme on peut l'observer sur les courbes de croissance (fig 1). Le poids final des 2 lots d'animaux s'avère identique (environ 250 g). L'efficacité alimentaire est inchangée (fig 2).

Cependant, on observe une modification du poids de certains tissus (fig 2). II se 


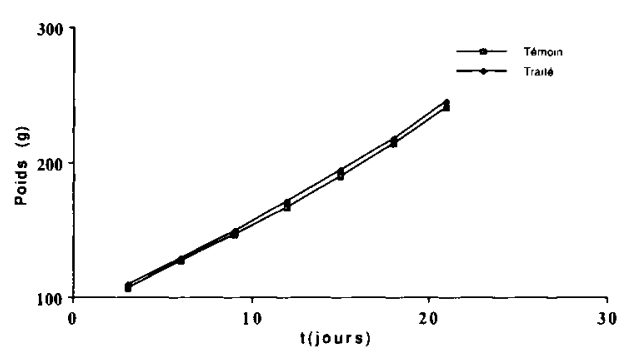

Fig 1. Courbes de croissance.

produit une augmentation, par rapport au groupe témoin, du poids du muscle gastrocnémien $(P<0,05)$ et du muscle cardiaque $(P<0,01)$. Par contre, une diminution du poids des tissus adipeux scapulaire et périrénal $(P<0,01)$ est observée.

Le traitement produit un accroissement de la masse protéique $(P<0,05)$ associé à une augmentation de la masse hydrique $(P<0,05)$ et à une diminution de la graisse $(P<0,01)$. La quantité de minéraux des carcasses reste identique dans les 2 lots d'animaux. On note également une diminu- tion nette $(P<0,001)$ de la quantité de glycogène dans le foie des animaux traités (tableau I).

La réduction de la masse produite par le métaprotérénol s'accompagne d'une augmentation de la consommation d'oxygène dans le tissu adipeux scapulaire $(P<0,05)$. Par contre, cet effet n'est pas retrouvé dans le muscle squelettique. La consommation d'oxygène de l'animal entier n'est pas modifiée d'une façon statistiquement significative (tableau II).

Le traitement produit une très forte augmentation de la quantité d'ADN du muscle gastrocnémien $(P<0,001)$ sans modification de la quantité d'ARN (tableau III).

Le processus de synthèse protéique ne semble pas être modifié puisque les paramètres qui le représentent (ARN/protéine, $K_{s}$ et $K_{A R N}$ ) sont identiques dans les deux groupes d'animaux (tableau III). On observe une diminution de l'activité de la cathepsine A musculaire chez les animaux traités $(P<0,001)$ et donc, dans le taux de dégradation protéique par jour $(K d ; \% / j)$ (tableau III). On trouve également une augmentation significative $(P<0,05) \mathrm{du}$

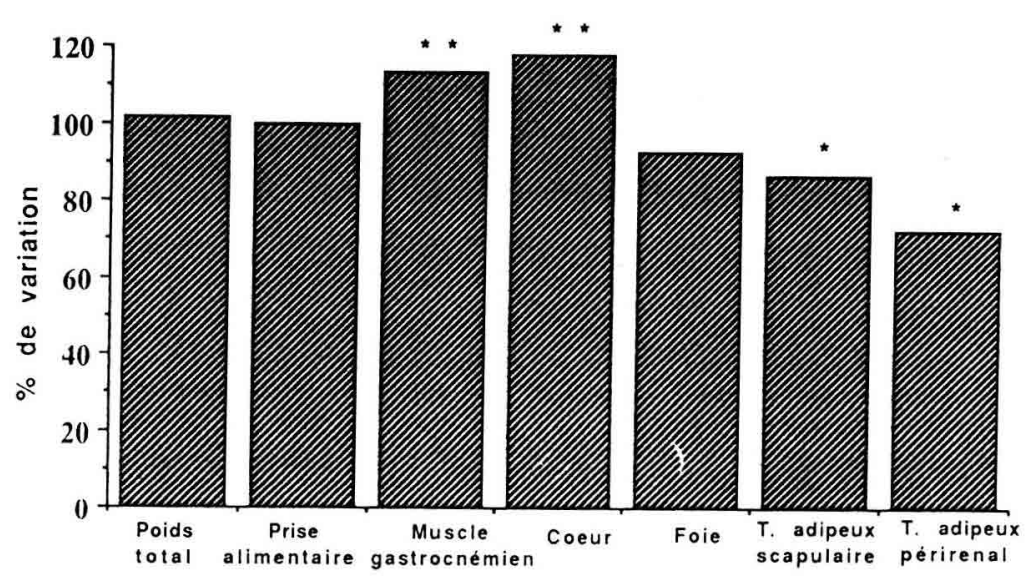

Fig 2. Pourcentage de modification du poids final des animaux, de l'efficacité alimentaire et du poids de quelques organes et tissus (muscle gastrocnémien, cœur, foie, tissu adipeux scapulaire et tissu adipeux périrénal) dans le groupe traité par rapport au groupe témoin $(100 \%)(n=8)$. 
Tableau I. Composition corporelle et contenu hépatique en glycogène .

\begin{tabular}{lrrr}
\hline & \multicolumn{1}{c}{ Témoin } & \multicolumn{1}{c}{ Traité } & \\
\hline Composition corporelle & & & \\
Protéine (\%) & $19,9 \pm 0,19$ & $20,9 \pm 0,39$ & $*$ \\
Graisse (\%) & $6,2 \pm 0,27$ & $3,1 \pm 0,27$ & $*$ \\
Eau $(\%)$ & $65,0 \pm 0,69$ & $66,9 \pm 0,68$ & $*$ \\
Minéraux (\%) & $3,2 \pm 0,12$ & $3,3 \pm 0,04$ & \\
Glycogène hépatique $(\mu g / g)$ & $45,4 \pm 2,11$ & $33,7 \pm 0,86$ & $*$ \\
\hline
\end{tabular}

$n=8 ; * P<0,05 ; * * P<0,01 ; * * * P<0,001$.

Tableau II. Consommation d'oxygène de l'animal entier et de quelques tissus : muscle gastrocnémien et tissu adipeux scapulaire.

\begin{tabular}{lcc}
\hline & Témoin & Traité \\
\hline $\begin{array}{l}\text { Animal entier } \\
\mathrm{ml} \text { (oxygène). } \mathrm{g}^{-1} \text { (poids corporel). } \mathrm{h}^{-1}\end{array}$ & $0,38 \pm 0,04$ & $0,40 \pm 0,02$ \\
$\begin{array}{l}\text { Muscle gastrocnémien } \\
\text { (nmol oxygène/100 mg tissu frais par min) }\end{array}$ & $14,07 \pm 0,78$ & $15,14 \pm 0,78$ \\
$\begin{array}{l}\text { Tissu adipeux scapulaire } \\
\text { (nmol oxygène/100 } \mathrm{mg} \text { tissu frais par min) }\end{array}$ & $21,26 \pm 1,39$ & $25,15 \pm 1,37^{*}$ \\
\hline
\end{tabular}

$n=8 ; * P<0,05$.

Tableau III. Acides nucléiques (ADN et ARN), activité de la cathepsine $A$ et paramètres indicateurs des processus de synthèse et de dégradation des protéines dans le muscle gastrocnémien. Le $\mathrm{Kg}$ représente le pourcentage de croissance musculaire par jour, le Ks le pourcentage de synthèse protéique par jour,le $K d$ le pourçentage de dégradation protéique par jour, et le $K_{A R N}$ l'activité de synthèse protéique.

\begin{tabular}{|c|c|c|c|c|c|c|c|}
\hline & $\vdots$ & & Témoin & & Traité & & \\
\hline $\begin{array}{l}\text { ADN/g (mg/g) } \\
\text { ARN/g (mg/g) } \\
\text { ARN/protéine ( } \mu \mathrm{g} / \mathrm{g}) \\
\mathrm{Kg}(\% / \mathrm{jour}) \\
\mathrm{Ks}(\% / \mathrm{jour}) \\
K_{\text {ARN }}(\mathrm{g} \text { prot/synth/g ARN par jc } \\
\text { Cathepsine A (unités/mg ADN) } \\
\text { Kd (\%/jour) }\end{array}$ & $\begin{array}{l}\cdot \\
\vdots \\
i \\
\text { our) } \\
\ldots \\
\ldots\end{array}$ & $\begin{array}{l} \\
13,4 \\
\cdots\end{array}$ & $\begin{aligned} & 0,52 \pm 0,02 \\
& 1,20 \pm 0,05 \\
& 5,9 \pm 0,3 \\
& 4,7 \pm 0,1 \\
& 8,1 \pm 0,2 \\
& \pm 0,3 \quad 14,6 \pm 0,8 \\
& 157,6 \pm 6,5 \\
& 3,4 \pm 0,2 \\
& \quad\end{aligned}$ & 8 & $\begin{array}{c}0,78 \pm 0,05 \\
1,19 \pm 0,04 \\
5,8 \pm 0,3 \\
5,6 \pm 0,4 \\
8,2 \pm 0,5 \\
93,8 \pm 8,1 \\
2,6 \pm 0,2\end{array}$ & $\begin{array}{l}* * * \\
*\end{array}$ & \\
\hline
\end{tabular}


taux de croissance musculaire $(\mathrm{Kg} ; \% / \mathrm{j})$ (tableau III).

Dans le groupe traité, on observe une augmentation du taux de prostaglandine $E_{2}(P<0,05)$ et du rapport lactate/pyruvate $(P<0,01)$ dans le muscle gastrocnémien (fig 3). Finalement, l'activité de l'ATPase $\mathrm{Ca}^{2+}-\mathrm{Mg}^{2+}$ n'apparaît pas significativement modifiée par le traitement (fig 3).

\section{DISCUSSION}

Les travaux publiés jusqu'à présent dans ce domaine de recherche ont été réalisés avec des agonistes $\beta_{2}$-sélectifs (clenbutérol, cimatérol, etc) (Eisemann et al, 1988; Walker et al, 1989). Traditionnellement, les effets lipolytiques ont été reliés à la présence de récepteurs $\beta_{t}$-adrénergiques (Ariens et Simons, 1983). Plus récemment, l'activité lipolytique de certains produits connus comme des agonistes $\beta$-atypiques a été démontrée (Bojanic et al, 1985; Granneman, 1990).

L'idée d'observer si, avec un agoniste $\beta_{1}-\beta_{2}$-adrénergique, on obtenait des effets lipolytiques plus puissants qu'avec les agonistes $\beta_{2}$-sélectifs utilisés jusqu'à présent, conservant toujours les effets sur les protéines, justifie le choix du métaprotérénol.

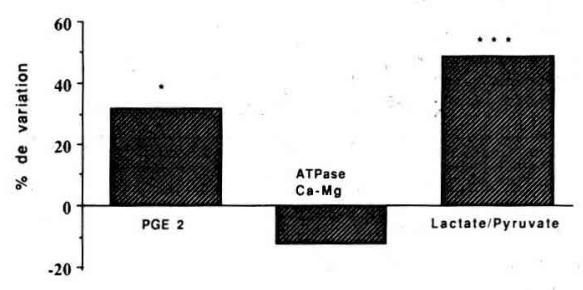

Fig 3. Pourcentage de modification de quelques paramètres potentiellement reliés au processus de dégradation protéique (prostaglandine $E_{2}$, ATPase $\mathrm{Ca}^{2+}-\mathrm{Mg}^{2+}$ et relation lactate/pyruvate) dans le groupe traité par rapport au groupe témoin $(n=8 ; " P<0,05 ; \cdots * P<0,001)$.
Les courbes correspondant aux 2 groupes d'animaux sont parallèles, traduisant l'absence de modification du taux de croissance, liée au traitement. II n'existe pas de différence sur la prise alimentaire, fait extrêmement important dans le cadre de l'utilisation de ces produits en production animale (Ferrando et Vanbelle, 1989; Buttery et Dawson, 1990; Maltin et al, 1990).

Après traitement par le métaprotérénol, on constate une augmentation du poids $\mathrm{du}$ muscle gastrocnémien et du cœur et une diminution du tissu adipeux scapulaire et périrénal. Ces résultats vont dans le même sens que ceux publiés à partir d'autres travaux employant le clenbutérol chez plusieurs espèces (Ricks et al, 1984; Reeds et al, 1986; Miller et al, 1988).

Tous ces résultats sont en accord avec les résultats obtenus lors de l'étude de la composition des carcasses. Le métaprotérénol s'avère être un agent "répartiteur", capable de modifier la relation protéine/ graisse/eau. On observe donc un accroissement de la masse protéique, accompagné d'une augmentation de l'eau et d'une diminution des graisses. Nous avons mesuré l'effet glycogénolytique du produit. Effectivement, on peut apprécier dans le foie des animaux traités une plus faible quantité de glycogène que dans celui des animaux témoins. Cet effet est lié à l'activation de l'enzyme glycogène-phosphorylase par l'augmentation de l'AMP ${ }_{c}$ induite par le métaprotérénol (Lafontan et al, 1988).

II est bien connu que les $\beta$-agonistes agissent comme des "répartiteurs" et qu'ils seraient capables de modifier le métabolisme énergétique, en activant la thermogenèse dans le tissu adipeux brun (Rothwell et Stock, 1987). Ce tissu est le principal responsable de la thermogenèse (Landsberg et al, 1984; Astrup et al, 1985). Dans notre cas, nous observons que le $\beta$ agoniste est capable de produire une sti- 
mulation de ce processus car il augmente la consommation d'oxygène dans ce tissu.

Certains auteurs considèrent aussi le muscle squelettique comme un endroit important où aurait lieu le phénomène thermogénique, après l'administration des agents adrénergiques (Thurlby et Ellis, 1986). On n'a pas trouvé de différence significative pour la consommation d'oxygène du muscle gastrocnémien. De même, ce produit ne modifie pas la consommation d'oxygène de l'animal entier. II faut remarquer que ce n'est pas le métabolisme de base mais celui de repos qui a été mesuré.

On a envisagé l'étude de l'action du métaprotérénol sur les protéines qui sont soumises constamment à des processus de synthèse et de dégradation, dans un équilibre dynamique (Waterlow et al, 1978). Les déterminations des acides nucléiques apportent des informations à propos des phénomènes d'hyperplasie (prolifération cellulaire) ou d'hypertrophie (accroissement de la taille cellulaire). La quantité d'ADN est un index du nombre de cellules d'un tissu (Burleigh, 1980). Par contre, la quantité d'ARN est représentative du processus de synthèse; plus précisément, la relation ARN/protéine est une mesure indirecte de la capacité de synthèse, c'est-àdire de la quantité d'outils biosynthétiques, mais elle n'informe pas sur l'activité de synthèse (Waterlow et al, 1978).

Les résultats obtenus semblent indiquer que le traitement avec le métaprotérénol produit un phénomène d'hyperplasie à cause de l'augmentation de la quantité d'ADN observée chez les animaux traités. Une incorporation de cellules satellites pourrait expliquer ce fait, car ces cellules sont les précurseurs de myocytes et sont capables de déclencher une croissance embryonnaire et donc proliférative (Burleigh, 1974, 1980). En ce qui concerne
I'ARN et la relation ARN/protéine, les résultats montrent que la synthèse n'est pas apparemment impliquée dans la modification du métabolisme protéique observée.

Tous ces paramètres ne sont que des indicateurs indirects. C'est pour cela qu'une détermination directe de la synthèse a été faite en employant la technique de Garlick et al (1980), qui définit le pourcentage de synthèse protéique par jour. Les données indiquent que ce processus n'est pas modifié par le traitement. Cependant, même si la capacité de synthèse n'est pas altérée, on pouvait espérer une modification de l'activité synthétique. Les valeurs observées de $K_{A R N}$ ne confirment pas cette hypothèse.

L'activité d'une enzyme protéolytique, la cathepsine $A$, un index de la dégradation protéique dans le muscle, a été considérée (Obled et al, 1980). L'activité de cette enzyme était plus faible chez les animaux traités. On peut donc conclure que l'accroissement de la masse protéique produit par le métaprotérénol serait dû à une diminution de la dégradation protéique. Des résultats similaires sont rencontrés dans la littérature (Morgan et al, 1986; Kretchmar et al, 1990).

L'implication de certains médiateurs dans le mécanisme d'action des agonistes $\beta$-adrénergiques peut être envisagée. D'après plusieurs auteurs (Tischler et Fagan, 1982; Chua et Kleinhans, 1985; Millward, 1985), il existe une relation entre l'état redox et le métabolisme des protéines. Dans les situations de catabolisme rapide, l'état redox serait plus oxydé, tandis que dans celles de catabolisme ralenti, l'état redox serait plus réduit (Oddy et al, 1987). La relation lactate/pyruvate est un témoin du rapport NADH/NAD et donc de l'état redox (Hedden et Buse, 1982). Cette relation augmente de façon prononcée dans les muscles des rats traités où l'on 
avait observé une activité plus faible de la cathepsine $A$. L'état redox réduit et la diminution de la dégradation protéique seraient donc corrélés.

Un autre médiateur pourrait intervenir : la prostaglandine $E_{2}$. Une augmentation des taux de cette prostaglandine s'observe dans les muscles traités, parallèlement à une diminution des taux de dégradation protéique. La littérature rapporte des travaux confirmant nos résultats, par exemple la stimulation de la synthèse de prostaglandines produite par l'AMP (Van den $_{c}$ Bosch, 1980). Or, nous savons que les agonistes $\beta$-adrénergiques activent l'adénylate-cyclase et provoquent une augmentation de l'AMP ${ }_{\mathrm{c}}$ (Stiles et al, 1984).

À partir de ces résultats, on peut conclure que le métaprotérénol, un agoniste $\beta_{1}-\beta_{2}$-adrénergique qui n'a jamais été employé pour ses effets sur le métabolisme, soit dans le domaine clinique, soit dans le domaine de la production animale, s'avère être un agent «répartiteur», capable de modifier le rapport protéine/ graisse/eau de l'organisme. L'accroissement protéique est dû à une diminution de la dégradation des protéines sans modification du processus de synthèse. Les modifications du métabolisme protéique sont corrélées aux différences de l'état redox et dés taux de prostaglandine $\mathrm{E}_{2}$.

\section{REMERCIEMENTS}

Les auteurs remercient l'aide économique du gouvernement de Navarra (1542/87) et du ministère de l'Éducation et de la Science (CICYT). Le $\beta$-agoniste a été fourni gratuitement par Boehringer-Ingelheim (Barcelone, Espagne).

\section{RÉFÉRENCES}

AOAC (1986) Official methods of analysis, 12th edn. Association of Official Analytical Chemists, Washington)
Ariens EJ, Simons AM (1983) Physiological and pharmacological aspects of adrenergic receptor classification. Biochem Pharmacol 32, 1539-1545

Astrup A, Bullow J, Christiensen NJ, Nadsen J, Quaade $F$ (1985) Facultative thermogenesis induced by carbohydrate: a skeletal muscle component mediated by epinephrine. Am J Physio/ 250, E226-E229

Babij P, Booth FW (1988) Clenbuterol prevents or inhibits loss of specific mRNAs in atrophying rat skeletal muscle. Am J Physiol 254, C657-C660

Baker $P$, Dalrympe $R H$, ingle $D L$, Ricks $C A$ (1984) Use of $\beta$-adrenergic agonist to alter muscle and fat deposition in lambs. J Anim Sci 59, 1256-1261

Bojanic D, Jansen J, Nahorski SR, Zaagsma J (1985) Atypical characteristics of the $\beta$ adrenoceptor mediating cyclic AMP generation and lipolysis in the rat adipocyte. $\mathrm{Br} J$ Pharmacol 84, 131-137

Burleigh IG (1974) On the cellular regulation of growth and development in skeletal muscle. Biol Rev 49, 267-319

Burleigh IG (1980) Growth curves in muscle nucleic acid and protein: problems in interpretation at the level of the muscle cell. In: Growth in animals (HN Munro, ed) Butterwoths, London, 101-106

Buttery PJ, Dawson JM (1986) The mode of action of beta-agonists as manipulators of carcass composition. In: Beta-agonists and their effects on animal growth and carcass quality (JP Hanrahan, ed) Elsevier Applied Science, Brussels, 29-43

Buttery PJ, Dawson JM (1990) Growth promotion in farm animals. Proc Nutr Soc 49, 459466

Chua BHL, Kleinhans BJ (1985) Effect of redox potential on protein degradation in perfused rat heart. Am J Physio/ 248, 726-731

Eisemann JH, Hungington GB, Ferrel CL (1988) Effects of dietary clenbuterol on metabolism of the hindquarters in steers. J Anim Sci 66, 342-353

Fain JN, Garcia-Sainz J (1983) Adrenergic regulation of adypocite metabolism. J Lipid Res 42, 945-966

Ferrando $R$, Vanbelle $M$ (1989) $\beta$-agonistes et production de la viande. Considerations et réflexions. Rec Med Vet 165, 91-96 
Fiems LO (1987) Effect of beta-adrenergic agonists in animal production and their mode of action. Ann Zootech 36, 271-290

Fiske $\mathrm{CH}$, Subbarow YJ (1925) The colorimetric determination of phosphorus. $J$ Biol Chem 66, 375-400

Garlick PJ, McNurland MA, Preddy UR (1980) A rapid and convenient technique for measuring the rate of protein synthesis in tissues by injection of $\left[{ }^{3} \mathrm{H}\right]$ phenylalanine. Biochem $J$ $192,719-723$

Granemann JG (1990) Norepinephrine and BRL 37344 stimulate adenylate cyclase by different receptors in rat brown adipose tissue. $J$ Pharmacol Exp Ther 254, 508-513

Hamby PL, Stouffer JR, Smith SB (1985) Muscle metabolism and carcass traits in lambs fed diets containing a beta-agonist. $J$ Anim Sci 61 (Suppl 1), 246 (abstr)

Hedden MP, Buse MG (1982) Effects of glucose, pyruvate, lactate and aminoacids on muscle protein synthesis. Am J Physiol 242, E184-E192

Kretchmar DH, Hataway MR, Epley RJ, Dayton WR (1990) Alterations in post-mortem degradation of myofibrillar proteins in muscle of lambs fed a $\beta$-adrenergic agonist. J Anim Sci 67, 2646-2654

Lafontan M, Berlan M, Prud'hon M (1988) Les agonistes bêta-adrénergiques. Mécanismes d'action : lipomobilisation et anabolisme. Reprod Nutr Dev 28, 61-84

Landsberg L, Saville ME, Young JB (1984) Sympathoadrenal system regulation of thermogenesis. Am J Physiol 247, E181-E189

Levine L, Alam I (1979) Arachidonic acid metabolism by cells in culture: analyse of culture fluids for cyclooxigenase products by radioimmuno-assay before and after separation by high pressure liquid chromatography. Prostaglandins 3, 297-304

MacRae JC, Lobley GE (1991) Physiological and metabolic implications of conventional and novel methods for the manipulation of growth and production. Livestock Prod Sci 27, 43-60

Maltin CA, Delday MI, Hay SM, Innes GM, Williams PEV (1990) Effects of bovine pituitary growth hormone alone or in combination with the $\beta$-agonist clenbuterol on muscle growth and composition in veal calves. $\mathrm{Br} J$ Nutr 63, 535-554

Martinez JA (1987) Validation of a fast, simple and reliable method to assess protein synthesis in individual tissues. I Biochem Biophys Methods 14, 349-354

Martinez JA, Goena M, Santidrian S, Larralde J (1987) Response of muscle, liver and muscle body protein turnover to two different sources of protein in growing rats. Ann Nutr Metab 31, 146-153

Miller MF, Garcia DK, Coleman ME, Ekeren PA, Lunt DK, Wagner KA, Procknor M, Walsh TH, Smith SB (1988) Adipose tissue, longuissimus muscle and anterior pituitary growth and function in clenbuterol-fed heifers. $J$ Anim Sci 66, 12-20

Millward DJ (1985) The physiological regulation of proteolysis in muscle. Biochem Soc Trans 13, 1023-1026

Morgan HE, Chua BHL, Watson PA, Russo L (1986) Protein synthesis and degradation. In: The heat and cardiovascular system (HA Fozzard, ed) Raven Press, New-York, 153178

Moser RL, Dalrymphe RH, Cornelius SG, Pettigrew JE, Allen CE (1986) Effect of cimaterol ( $\mathrm{CL} 263^{\prime} 780$ ) as a repartitioning agent in the diet for finishing pigs. J Anim Sci 62, 21-26

Obejero CA, Gonzales DA, Alonso GL (1988) Demonstration of the simultaneous activation

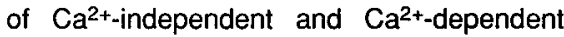
ATPase from rat skeletal muscle microsomes. Biochim Biophys Acta 939, 409-415

Obled C, Arnal M, Valin C (1980) Variation through the day of hepatic and muscular cathepsin $A, C$ and $E$ activities and amino acids of blood in rats: influence of feeding schedule. Br J Nutr 44, 61-69

Oddy VH, Lindsay DB, Baker PJ, Horthrop AJ (1987) Effect of insulin on hind-limb and whole body leucine and protein metabolism in fed and fasted lambs. Br J Nutr 58, 437452

Reeds PJ, Hay SM, Dorwood M, Palmer RM (1986) Stimulation of muscle growth by clenbuterol: lack of effect on muscle protein biosynthesis. Br J Nutr 56, 249-258

Ricks CA, Muir LA, Duquette PF (1985) Effect of beta-adrenergic agonist $L-640^{\prime} 033$ on growth 
and carcass composition of growing male rats. J Anim Sci 61 (Suppl 1), 264 (abstr)

Rothwell NJ, Stock MJ (1987) Influence of clenbuterol on energy balance, thermogenesis and body composition in lean and genetically obese Zucker rats. Int J Obesity 11, 641-647

Rothwell NJ, Stock MJ, Sudera DK (1987) Changes in tissu blood flow and $\beta$-receptor density of skeletal muscle in rats treated with the $\beta_{2}$-adrenoceptor agonist clenbuterol. $\mathrm{Br} J$ Pharmacol 90, 601-607

Simonides WS, Van Hardeveld C (1988) $\left(\mathrm{Ca}^{2+}\right.$ $\left.\mathrm{Mg}^{2+}\right)$ ATPase activity associated with the maintenance of a $\mathrm{Ca}^{2+}$ gradient by sarcoplasmic reticulum at micromolecular external $\left[\mathrm{Ca}^{2+}\right.$. The effect of hypothyroidism. Biochim Biophys Acta 943, 349-359

Stiles GL, Caron MG, Lefkowitz RJ (1984) $\beta$ adrenergic receptors: biochemical mechanisms of physiological regulation. Physiol Rev 64, 661-735

Suzuki O, Yagi K (1976) A fluorimetric assay for $\beta$-phenylalanine in rat brain. Anal Biochem $75,192-200$

Thurlby PL, Ellis DM (1986) Differences between the effects of noradrenaline and the $\beta$-adrenoceptor BRL 26830A. Can J Physiol Pharmacol 65, 113-123

Timmerman $H$ (1987) $\beta$-adrenergics: physiology, pharmacology, applications, structures et structure-activity relationships. In: Beta- agonists and their effects on animal growth and carcass quality (JP Hanrahan, ed) Elsevier Appl Sci, London, 13-28

Tischler ME, Fagan JM (1982) Relationship of the reduction-oxidation state to protein degradation in skeletal and atrial muscle. Arch Biochem Biophys 217, 191-201

Van den Bosch $H$ (1980) Intracellular phosphorilases A. Biochim Biophys Acta 604, 191-246

Vidaver $W$, Swenson $S$ (1989) $\mathrm{O}_{2}$ Exchange measurement using a platinum polarographic electrode. In: Gases in plant and microbial cells (HF Linskens, ed) Springer-Verlag, Berlin, 41-61

Walker WR, Johnson DD, Brendemuhl JH, Dalrymphe RH (1989) Evaluation of cimaterol for finishing swine including a drug withdrawal period. J Anim Sci 67, 168-176

Warner C, Colina I, Warner F, Corto J, Prieto J, Vilardell $F$ (1986) Renal prostaglandins in cyrrhosis of the liver. Clin Sci 70, 477-484

Waterlow JC, Garlick PJ, Millward DJ (1978) The effect of nutrition and hormones on protein turnover in muscle. In: Protein turnover in mammalian tissues and in the whole body (JC Waterlow, ed) North-Holland Publishing Company, Amsterdam, 698-746

Zeman RJ, Ludemann R, Etlinger JD (1987) Clenbuterol, a $\beta_{2}$-agonist, retards atrophy in denervates muscles. Am J Physiol 252, E152-E155 